\title{
Cefprozil Anhydrous, (Z)-
}

National Cancer Institute

\section{Source}

National Cancer Institute. Cefprozil Anhydrous, (Z)-. NCI Thesaurus. Code C80091.

The anhydrous form of the Z-isomer of a semi-synthetic cephalosporin and a betalactam antibiotic with bactericidal activity. Cefprozil's effect is dependent on its binding to penicillin-binding proteins (PBPs) located in the bacterial cytoplasmic membrane. Binding results in the inhibition of the transpeptidase enzymes, thereby preventing cross-linking of the pentaglycine bridge with the fourth residue of the pentapeptide and interrupting consequent synthesis of peptidoglycan chains. As a result, cefprozil inhibits bacterial septum and cell wall synthesis formation. 